\title{
Selenium yeast: Composition, quality, analysis, and safety*
}

\author{
G. N. Schrauzer \\ Department of Chemistry and Biochemistry, University of California, San Diego, \\ and Biological Trace Element Research Institute, Chula Vista, CA 91914, USA
}

\begin{abstract}
Selenium yeast, produced by growing select strains of Saccharomyces cerevisiae in Se-rich media, is a recognized source of organic food-form Se, but the determination of its exact composition with respect to the Se species present produced conflicting results. Improved methods of analysis have since revealed it to contain $90+\%$ of its Se in the form of selenomethionine, the principal organic nutritional form of Se for higher animals and humans. The safety record of Se yeast is excellent: During the three decades of its world-wide use as a source of supplemental Se, no cases of Se poisoning have occurred due to dosage or formulation errors.
\end{abstract}

Keywords: selenium; selenium yeast; Saccharomyces cerevisiae; selenomethionine; supplemental selenium; selenium poisoning.

\section{INTRODUCTION}

In 1957, K. Schwarz and W. Foltz [1] showed that Se was an integral component of a factor isolated from yeast ("Factor 3") which prevented dietary liver necrosis in rats. Schwarz recognized that Factor 3 was an organic rather than inorganic Se compound, but was unable to identify it. It may be concluded that Factor 3 probably consisted of selenomethionine, the major naturally occurring Se compound in yeast [2]. Since Factor $3 \mathrm{Se}$ was not available and a source of Se was urgently needed to combat Se deficiency in farm animals, sodium selenite and -selenate were approved in the early 1970s as feed additives by the U.S. Food and Drug Administration. The recognition that these inorganic Se salts were generally less effective than Factor 3 Se prompted Universal Foods, then America's leading producer of food yeast, on initiative of Nutrition 21, a California nutritional supplement company, to develop a process for producing Se-enriched yeast as a source of Factor $3 \mathrm{Se}$. The first batches of Se yeast reached the marketplace in 1974. Today, Se yeast is produced by several manufacturers and has become the most widely used source of Se for human supplementation. Selenium yeast is also increasingly employed in animal nutrition, its use is FDA-approved for several animal species. Although Se yeast has been produced by standardized methods with well-developed quality controls for three decades, some authors recently questioned its quality and safety, arguing that its composition with respect to the Se compounds present was inconstant, and that a significant percentage of its Se compounds were as yet unidentified. As a result of these claims, the European Community's Scientific Committee on Food concluded that Se yeast was too poorly characterized for use in food supplements [3]. In addition, the Committee felt that, because of its high selenomethionine content, the ingestion of Se yeast could result in the accu-

\footnotetext{
*Paper based on a presentation at the $2^{\text {nd }}$ International Symposium on Trace Elements in Food (TEF-2), Brussels, Belgium, 7-8 October 2004. Other presentations are published in this issue, pp. 65-143.
} 
mulation of Se to toxic levels in organs and tissues. In consequence, the European Parliament and Council of the European Union (EU) in directive 2002/46/EC excluded Se yeast from its positive list on permitted food supplements. It will be shown in the following section that Se yeast is a reliable source primarily of selenomethionine, the principal nutritional form of Se for higher animals and humans, and that apparent inconsistencies or uncertainties of composition are caused more by analytical difficulties than actual variations of yeast quality. Finally, the safety of Se yeast will be discussed and whether the ingestion of selenomethionine results in its accumulation in the organism.

\section{SELENIUM YEAST MANUFACTURE}

Yeast (Saccharomyces cerevisiae) was reported as early as 1961 to take up inorganic Se from the culture medium and to convert it into selenomethionine [4]. The biosynthesis of selenomethionine is known to occur in analogy to that of methionine and to utilize the same enzymes [2]. The maximum amount of Se a yeast cell can theoretically incorporate accordingly depends on its methionine content and is in the order of $6000 \mathrm{ppm}$. However, the full replacement of methionine by selenomethionine is not possible - the highest amount of Se in yeast achieved so far is about $3000 \mathrm{ppm}$. The Se yeast of commerce is usually cultured to contain from 500 to $2000 \mathrm{ppm}$ of Se and is indistinguishable in appearance, flavor, odor, microbiological purity, and vitamin content from normal dried food yeast. Selenium yeast is produced industrially by methods which may differ in important details and which in part are proprietary and/or patented. For a description of some Se yeast production processes and suppliers, see [5-10]. To be marketable as a food, Se yeast must meet or exceed the standards laid down by IUPAC for dried food yeast. It is defined as "the whole organism of one individual yeast, or a mixture of several yeasts belonging to the family Saccharomycetaceae, obtained either as a by-product of fermentation processes or by special culture". Dried Se yeast must not exceed the upper limits of moisture $(10 \%)$, ash $(10 \%)$, lead $(5 \mu \mathrm{g} / \mathrm{g})$, arsenic $(5 \mu \mathrm{g} / \mathrm{g})$, live bacteria counts $(7500 / \mathrm{g})$, and mold counts $(50 / \mathrm{g})$. Minimum levels are set for nitrogen ( $7.2 \%$, equivalent to $45 \%$ protein), thiamine, riboflavin, and niacin. It is furthermore required to be free of starch and bacteria of the genus Salmonella.

\section{INORGANIC SELENIUM YEAST}

Whereas genuine Se yeast contains only traces of inorganic Se (less than $1 \%$ ), "inorganic" Se yeast preparations with added selenite or selenate where introduced into the market as competitive products during the early years of "organic" Se yeast development and are still offered by some companies. Since sodium selenite and -selenate are approved as supplemental forms of Se, inorganic Se yeast products are not in violation of regulations. However, the need for distinguishing them from genuine "organic" Se yeast has been emphasized [11-13].

\section{SELENIUM YEAST ANALYSIS}

The characterization of Se yeast requires the determination of selenomethionine and of all other major Se compounds present. In early Se speciation analyses using high-performance liquid chromatography methods, organoselenium compounds were determined in hot water yeast extracts. Since a significant percentage of selenomethionine in yeast is incorporated in the cellular protein it is not readily extractable. To obtain correct analytical results, the yeast proteins thus must be hydrolyzed either with acids or enzymatically, under conditions assuring complete hydrolysis with minimal decomposition of the organoselenium compounds present.

Yeast protein hydrolysis with acids requires vigorous conditions. To release the methionine in yeast proteins, Chiao and Peterson [14], for example, autoclaved the yeast sample at $120^{\circ} \mathrm{C}$ for $1 \mathrm{~h}$ with $7 \mathrm{~N}$ hydrochloric acid, $2 \mathrm{~h}$ with $5 \mathrm{~N}$ acid, or $3 \mathrm{~h}$ with $3 \mathrm{~N}$ acid. The recovery of methionine under these condition was $96 \%$ or better [12], but is lower for the less stable selenomethionine. To establish the op- 
timal hydrolysis conditions for Se yeast hydrolysis, trial runs with added known amounts of selenomethionine must be conducted. The hydrolysis furthermore must be conducted under exclusion of oxygen to minimize losses of selenomethionine through oxidation. Beilstein and Whanger in 1986 [15] hydrolyzed Se yeast in $6 \mathrm{~N} \mathrm{HCl}$ in an inert atmosphere and achieved selenomethionine recoveries of 55 to $85 \%$. Assuming an average recovery of $70 \%$, they calculated the SeMet content of Se yeast to $93 \%$ of the total Se present. Higher recoveries of selenomethionine are achieved with performic acid for yeast protein hydrolysis, followed by the reduction of the oxidized selenomethionine back to selenomethionine. With this method, the selenomethionine content of Se yeast was determined to $94 \pm 5 \%$ of total selenium [16], the highest percentage of selenomethionine measured in selenium yeast so far. Additional detected and identified Se compounds in this Se yeast included, in percentages of total Se: Se-adenosylselenohomocysteine $(2-5 \%)$, inorganic Se $(1 \%)$, traces of selenocystine, selenocystathionine, methylselenocysteine, and $\gamma$-glutamylselenocysteine, bringing the Se balance in terms of identified Se compounds to $100 \pm 5 \%[2,17]$.

Enzymatic yeast protein hydrolysis generally produces somewhat lower selenomethionine contents than acid hydrolysis, even with the most active, freshly prepared enzyme preparations. With Protease XIV (Sigma, St. Louis), a selenomethionine content of Se yeast of $85 \%$ of total Se was observed [18]. Other authors, as quoted in [10], observed selenomethionine contents of different batches or brands of yeast ranging from 81 to $84 \%$ of total Se, with 80 to $90 \%$ of the total Se accounted for. In another study [19], selenomethionine contents of Se yeast samples produced in the years 1985-2000 were reported. Observed selenomethionine contents in yeast samples produced from 1997-2000 ranged from $73.7 \pm 6.0$ to $81.0 \pm 5.6 \%$, with $0.2-0.8 \%$ of oxidized selenomethionine (SeOMet), $2-9 \%$ of "unknown" Se species, and 15-17\% of unaccounted (residual) Se. In one sample of Se yeast from 1985, the measured selenomethionine content was $30.0 \pm 2.5 \%$ of total Se, an additional $2.7 \pm 2.3 \%$ of Se was in the form of oxidized selenomethionine (SeOMet), $15.5 \%$ was designated as residual Se, and $\sim 51 \%$ of the total Se was in "unknown" chromatographic peaks. Unknown peaks in Se yeast hydrolysate chromatograms, in the present author's experience, are usually indicative of incomplete protein hydrolysis. It is possible that yeast proteins undergo irreversible changes during prolonged storage that render them more resistant to enzymatic hydrolysis. On prolonged storage, furthermore, new organoselenium compounds could be formed through secondary microbial or fungal action. One such compound could be the recently detected $S$-(selenomethyl)-cysteine [20]. It is concluded from this evidence that the observed differences of composition of stored Se yeast samples are more indicative of analytical problems than of variations of Se yeast quality.

\section{SELENOMETHIONINE IN HUMAN AND ANIMAL NUTRITION}

As a natural source of organic bioavailable food form of Se, Se yeast closely resembles seleniferous corn, wheat, and soybeans, whose SeMet contents reach 81-82\% of total Se [21]. Hence, selenomethionine is the form of Se in which it enters the food chain of higher animals and humans. Since higher animals and humans cannot synthesize selenomethionine, but from it all needed forms of Se are obtained, selenomethionine fulfills the criteria of an essential amino acid [2]. Selenomethionine furthermore can replace methionine in proteins, making it the only seleno-amino acid that can be significantly stored in organs and tissues. In humans, in absolute amounts, most of it is stored in the skeletal musculature. Other organic Se compounds that are found in certain plants such as methylselenocysteine, selenocystathionine, and $\gamma$-glutaminyl-Se-methylselenocysteine, cannot be stored in protein organs and tissues. These compounds are mainly found in primary Se accumulator plants and play no significant role as nutritional sources of Se [2,22]. 


\section{ACCUMULATION OF SELENOMETHIONINE IN ORGANS AND TISSUES}

Ingested selenomethionine is actively absorbed and is either metabolized directly or is incorporated into proteins. The metabolic breakdown of selenomethionine occurs in analogy to that of methionine, yielding selenocystine, which is metabolized further to selenide and either used for selenoprotein synthesis or methylated and excreted.

Can the prolonged ingestion of selenomethionine at nutritional levels result in the gradual accumulation of Se organs and tissues to toxic levels? The answer to this question is no, since mammalian enzymes do not to discriminate between selenomethionine and methionine. At constant intakes of both, the ratio of selenomethionine to methionine in proteins will remain constant, i.e., a steady state will establish itself. It is for this reason that a linear relationship exists between the dietary Se intakes and the Se levels in whole blood, which is obeyed over several orders of magnitude. The daily supplementation of Se in the form of Se yeast causes blood Se levels to increase for about 6 weeks. Thereafter, on continuing supplementation, blood Se levels begin to plateau, marking the beginning of the establishment of a steady-state condition [23]. Similarly, plasma Se levels of study subjects receiving $200 \mu \mathrm{g}$ of Se per day in the form of Se yeast increased during the first two months and henceforth remained constant for up to four years of supplementation [24]. Direct linear relationships also exist between the dietary Se intakes and the Se contents of hair, toenails, and fingernails [25]. While the rates at which steady-state conditions are reached in different organs may vary, there is evidently no uncontrolled accumulation of selenomethionine, even after prolonged supplementation at nutritional dosages.

\section{SELENIUM YEAST SAFETY}

Whereas pure selenomethionine is a toxic substance, $1 \mathrm{~g}$ of which taken orally could be fatal to an adult, Se yeast poses no comparatively serious threat since, to obtain the same amount of selenomethionine, approximately $1 \mathrm{lb}$ of a $1000 \mathrm{ppm} \mathrm{Se} \mathrm{Se}$ yeast would have to be ingested. The chronic toxicity of Se yeast has been reported to be lower compared to sodium selenite in experiments with weanling rats [26] and growing pigs [27]. However, this is true only for growing young animals, and not for older animals. Young, growing animals are less sensitive to chronically toxic levels of selenomethionine in the feed only because some of it is continuously removed from circulation and incorporated into newly synthesized proteins. Nevertheless, the use of Se yeast as a feed additive or source of Se for supplements is always safer than that of sodium selenite, if only because its low Se concentration safeguards against formulation or dosage errors. Accordingly, the safety record of Se yeast is excellent: In the three decades of its use as a feed additive and in nutritional supplements, no cases of accidental Se poisoning have been reported.

\section{CONCLUSION}

The first process of manufacturing Se yeast was developed more than three decades ago. Its chemical analysis, and especially the quantitative determination of selenomethionine, initially caused difficulties, resulting in claims that Se yeast was poorly characterized and of variable composition. In the meantime, the analytical problems have been largely overcome, and it is now generally agreed that Se yeast contains most of its total Se in the form of selenomethionine. The application of improved methods of analysis also revealed the composition of Se yeast to be more uniform than previously assumed. Since selenomethionine is the chief form of Se in which it enters the food chain, Se yeast thus is to be regarded as an excellent source of naturally biosynthesized, food-form Se.

Finally, concerns that the ingestion of selenomethionine may result in the accumulation of Se to potentially toxic levels in the organism must be judged as unfounded, since ingested selenomethionine is not only incorporated into proteins, but is also continuously released from them by normal catabolic processes. 


\section{REFERENCES}

1. K. Schwarz and C. M. Foltz. J. Am. Chem. Soc. 79, 3292 (1957).

2. G. N. Schrauzer. Adv. Food Nutr. Res. 47, 73 (2003).

3. EC Scientific Committee on Food (2003), Opinion of the Scientific Committee on Food on the Revision of Reference Values for Nutrition Labeling. Commission of the European Communities, Brussels (2003).

4. M. Blau. Biochim. Biophys. Acta 49, 389 (1961).

5. G. N. Schrauzer. J. Med. Foods 1, 201 (1998).

6. M. Korhola, A. Vainio, K. Edelmann. Ann. Clin. Res. 18, 65 (1986).

7. R. F. Power. In Proc. SDTA $5^{\text {th }}$ Internatl. Symp., pp. 89-94, 8-10 May, Brussels, STDA, Brussels.

8. A. Demirci, A. L. Pometto, D. J. Cox. J. Agric. Food Chem. 47, 2496 (1999).

9. A. Suhajda, J. Hegoczki, B. Janzso, I. Pais, G. Vereczkey. J. Trace Elem. Med. Biol. 14, 43 (2000).

10. M. Rayman. Br. J. Nutr. 92, 557 (2004).

11. G. N. Schrauzer and J. E. McGinness. Trace Subst. in Environ. Health XIII, 64 (1979).

12. W. Gössler, J. Zheng, W. Kosmus. In Spurenelemente-Speziationsanalysis, Supplementierung und Therapie mit Spurenelementen, D. Meißner (Ed.), pp. 61-64, Wissenschaftliche Verlagsgesellschaft mbH (1999).

13. G. N. Schrauzer. J. Am. Coll. Nutr. 20, 1 (2001).

14. A. S. Chiao and W. H. Peterson. Agric. Food Chem. 1, 1005 (1953).

15. M. A. Beilstein and P. D. Whanger. J. Nutr. 116, 1701 (1986).

16. G. N. Schrauzer. In Proc. Sixth Internatl. Symp. on the Uses of Selenium and Tellurium, Y. Palmieri (Ed.), pp. 77-79, 10-12 May 1998, Scottsdale, AZ.

17. G. N. Schrauzer. J. Nutr. 130, 1653 (2001).

18. C. Ip, M. Birringer, E. Block, M. Kotrebai, J. F. Tyson, P. C. Uden, D. J. Lisk. J. Agric. Food Chem. 48, 2062.

19. E. H. Larsen, M. Hansen, H. Paulin, S. Moesgaard, M. Reid, M. Rayman. J. AOAC Int. 87, 225 (2004).

20. E. Block, R. S. Glass, N. E. Jacobsen, S. Johnson, C. Kahakachi, K. Chethaka, R. Kaminski, A. Skowronska, B. T. Boakye, J. F. Tyson, P. C. Uden. J. Agric. Food Chem. 52, 3761 (2004).

21. X. Yang, Y. Tian, P. Ha, L. Gu. J. Hyg. Res. 26, 113 (1997).

22. N. Terry, A. M. Zayed, M. P. de Souza, A. S. Tarun. Ann. Rev. Plant Physiol., Plant Mol. Biol. 51, 401-432 (2000).

23. G. N. Schrauzer and D. A. White. Bioinorg. Chem. 8, 303 (1978).

24. L. C. Clark, G. F. Combs, B. W. Turnbull, E. H. Slate, D. K. Chalker, J. Chow, L. S. Davis, R. A. Glover, G. F. Graham, E. G. Gross, A. Krongrad, J. L. Lesher Jr., H. K. Park, B. B. Sanders Jr., C. L. Smith, J. R. Taylor. J. Am. Med. Assoc. 276, 1957 (1996).

25. G. Yang, R. Zhou, S. Yin, L. Gu, Y. Liu, X. Liu. J. Trace Elem. Electrolytes Health Dis. 3, 77 (1989).

26. J. E. Spallholz and A. Rafferty. In Selenium in Biology and Medicine, Part A, G. F. Combs, J. E. Spallholz, O. A. Levander, J. E. Oldfield (Eds.), pp. 516-529, Van Nostrand Reinhold, New York (1987).

27. Y. Y. Kim and D. C. Mahan. J. Anim. Sci. 79, 942 (2001). 